\title{
Electrically coupled MEMS Oscillators
}

\author{
Deepak K. Agrawal, Pradyumna Thiruvenkatanathan, Jize Yan and Ashwin A. Seshia \\ Department of Engineering, \\ University of Cambridge, \\ Cambridge CB2 1PZ, UK \\ Email: dka23@cam.ac.uk
}

\begin{abstract}
In this paper, we demonstrate synchronization of two electrically coupled MEMS oscillators incorporating nearly identical silicon tuning fork microresonators. It is seen that as the output of the oscillators are coupled, they exhibit a synchronized response wherein the output amplitudes and signal-to-noise ratios of the two oscillators are improved relative to the case where the two oscillators are uncoupled. The observed output frequency of each oscillator before coupling is 219402.4 Hz and 219403.6 $\mathrm{Hz}$ respectively. In contrast, when the oscillators are driven simultaneously, they lock at a common output frequency of $219401.3 \mathrm{~Hz}$ and their outputs are found to be out-of-phase with respect to each other. A $6 \mathrm{dBm}$ gain in output power and a reduction in the phase fluctuations of the output signal are observed for the coupled oscillators compared to the case when the oscillators are uncoupled.
\end{abstract}

\section{INTRODUCTION}

Over the last two decades, advancements in design, fabrication and packaging of silicon MEMS oscillators have resulted in their commercialization with the potential to replace quartz crystal oscillators from certain market sectors due to their smaller sizes, scalability to VHF-UHF frequencies and the potential for integration with CMOS electronics. However, MEMS oscillators are currently limited in their noise performance and output power capabilities, in part due to their smaller dimensions and the associated onset of non-linear self-limiting behaviour at relatively modest oscillation amplitudes.

Several methods have been previously employed to address these limitations. Of these, perhaps the most notable is the effort to enhance the noise performance of silicon MEMS oscillators by increasing the output power using array-coupled silicon microresonators [1]. In this work, an array of nine identically designed mechanically coupled wine-glass disk resonators operating in a combined resonant mode were embedded in an oscillator feedback loop to enhance the output motional current, thereby improving the phase noise performance. While it can be inferred from their results that the use of multiple resonators, enhances the oscillator response relative to the typical use of a single resonator, the use of multiple resonators also changes the modal dynamics, as each resonator adds an additional degree of freedom, making the system more susceptible to excitation of spurious modes. Moreover, electrostatic transduction of the resonator array in order to excite a particular combined vibration mode for an array coupled system can impose constraints on transducer geometry and routing interconnect as well as increase the effects of capacitive feedthrough parasitics.

Various noise sources with electrical, mechanical and environmental origins result in amplitude and phase fluctuations in the output of the MEMS oscillator [2]. Phase fluctuations are accumulated over time and are of particular interest in timing and frequency control applications [3]. In an attempt to address the phase stability of MEMS oscillators, we propose the utilization of the phenomenon of synchronization wherein synchronized oscillators lock at a common output frequency and a constant relative phase difference.

One of the first observations of synchronization was reported by the Dutch researcher Christiaan Huygens in the $17^{\text {th }}$ century. He observed that when two symmetric pendulum clocks were suspended from a common frame, they began to oscillate at a common frequency and out-of-phase with respect to each other due to the weak coupling that caused by the imperceptible motion of the frame [4].

More recently, Bennett et al. re-examined the phenomenon using two symmetric pendulum clocks and showed that the onset of synchronization was related to the extent of mismatch of their uncoupled oscillation frequencies and the strength of coupling between them [5]. The oscillation frequency for a MEMS oscillator is determined by the resonating element which is a MEMS resonator in our case. Tolerances in fabrication processes often result in variations in the dimensions of the resonator and in their material properties. Due to these variations, the nominal resonant frequencies across identically designed resonators are usually different. MEMS resonators can be coupled by mechanical or electrical methods [6]. As opposed to mechanical resonators in more conventional technologies, electrical coupling of MEMS resonators can be implemented by parallel-plate capacitive coupling elements that can be readily implemented in a standard micromachining process. In this work, the synchronization of two electrically coupled silicon MEMS oscillators is demonstrated. 


\section{DEVICE DESCRIPTION AND OPERATION}

\section{A. Device Configuration}

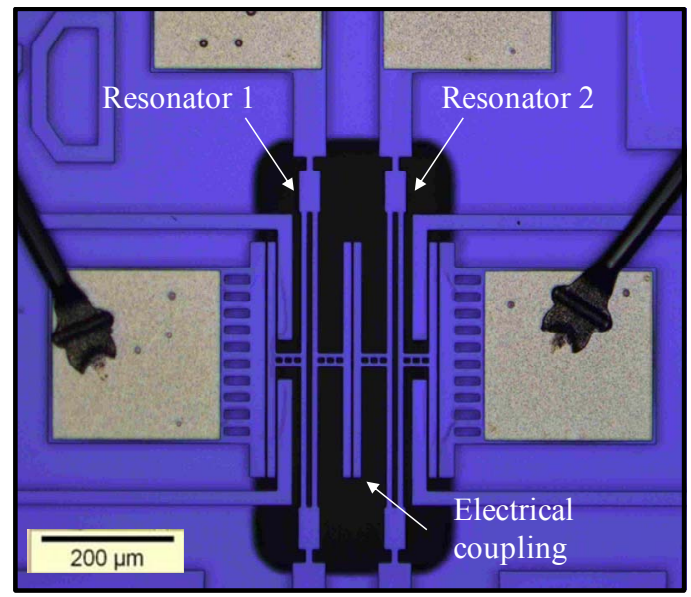

Figure 1. Optical micrograph of electrically coupled DETF resonators.

Fig. 1 shows an optical micrograph of two electrically coupled identically designed silicon double-ended-turningfork (DETF) resonators [6]. The device is fabricated in a commercial foundry process using the silicon-on-insulator MEMS process through MEMSCAP Inc., USA. The two resonators are separated by a coupling gap as can be seen in Fig. 1. In this device, each tine is $350 \mu \mathrm{m}$ long, $7 \mu \mathrm{m}$ wide and $25 \mu \mathrm{m}$ thick. Additional parallel plates are added at the point of maximum displacement to ensure uniform electrostatic actuation of the fundamental vibration mode. Each added electrode is $280 \mu \mathrm{m}$ long, $10 \mu \mathrm{m}$ wide and $25 \mu \mathrm{m}$ thick. All the actuation gaps in both the resonators are $2 \mu \mathrm{m}$ including the coupling gap. The out-of-phase normal mode of the resonator is of interest, as it offers a higher quality factor compared to the in-phase normal mode, due to the cancellation of stress at the anchor points. It is to be noted that any potential difference across the coupling gap generates an attractive electrostatic force which is displacement dependent and results in a negative mechanical spring like behaviour. The electrostatic coupling force $\left(F_{c}\right)$ between two resonators can be determined by:

$$
F_{c}=-\frac{\varepsilon_{o} A}{d^{2}}\left(V_{D C 1}-V_{D C 2}\right)^{2}
$$

Here $V_{D C 1}$ and $V_{D C 2}$ are the $D C$ bias applied to each of the resonators. $A$ is the actuation area, $d$ is actuation gap and $\varepsilon_{o}$ is permittivity of free space. It can be deduced from equation (1) that the change in potential difference across these resonators results in a modified coupling force.

\section{B. Device Characterization}

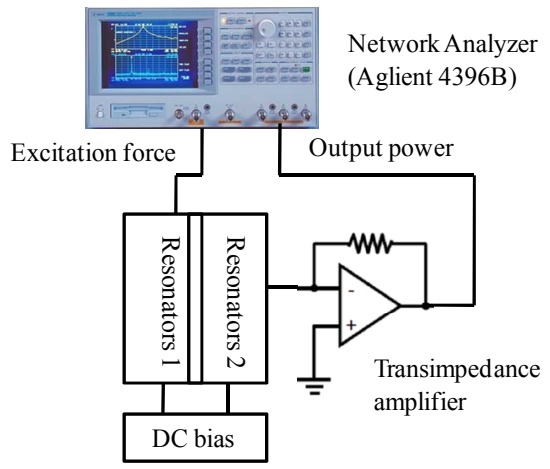

Figure 2. Schematic representation of open loop measurement setup for silicon DETF MEMS resonators.

A schematic representation of the measurement setup for open-loop experiments is shown in Fig. 2. The device is tested in a custom vacuum chamber at a pressure of $\approx 50$ mtorr. Two-port electrostatic transduction is employed to detect the motion of the resonators. An $A C$ excitation force is applied to the network analyzer to drive the resonators while $D C$ bias is used to detect the motional current. The output motional current is amplified and converted to a voltage by a transimpedance amplifier and then sent to the network analyzer.

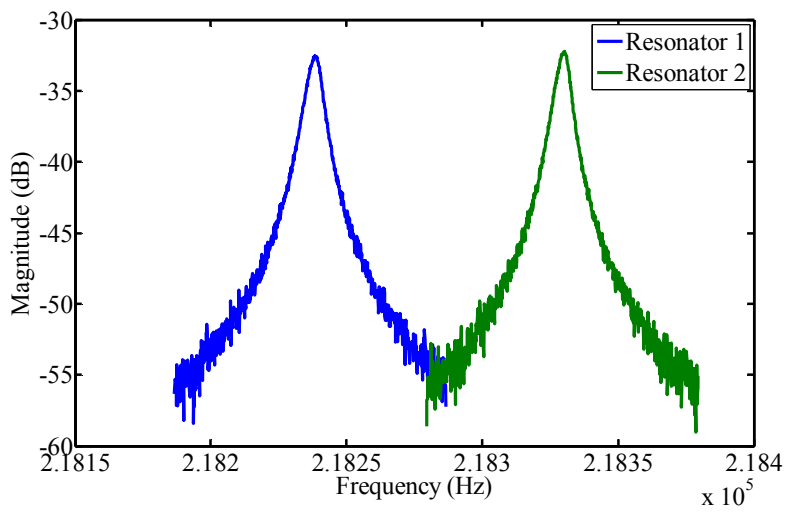

Figure 3. Open loop response of uncoupled DETF MEMS resonators.

The result of the measurements for the uncoupled response after feedthrough cancellation is shown in Figure 3. The feedthrough component is obtained by applying $0 \mathrm{~V}$ potential difference across the actuation gap. The quality factor and resonance frequency for each resonator are 31177, 32956 and $218238.3 \mathrm{~Hz}, 218330.0 \mathrm{~Hz}$ respectively. These values are determined by a nyquist circle fit to the measurements of the uncoupled response. Although, the resonators are designed to be identical, fabrication tolerances result in variations in dimensions and material properties, consequently leading to deviations in the resonance frequencies for identically 


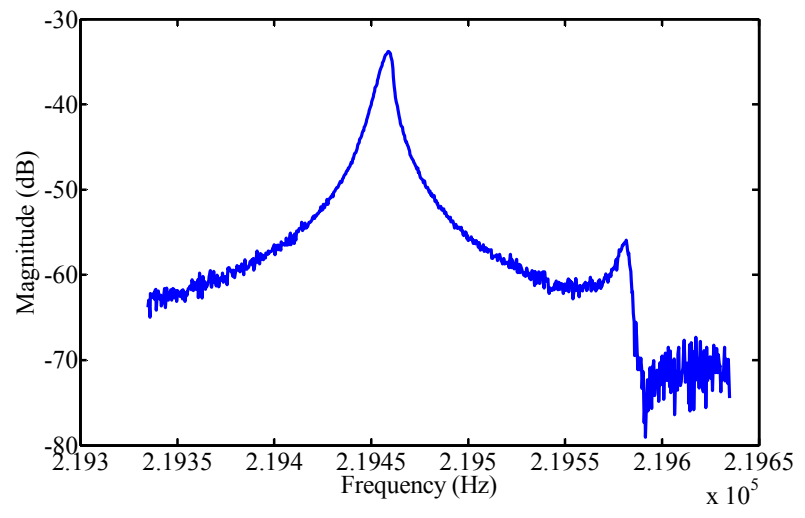

(a)

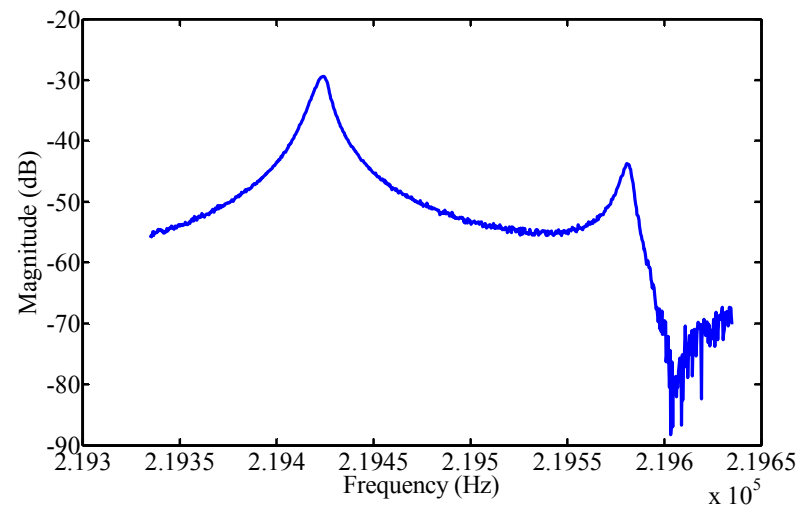

(b)

Figure 4. Open loop response of coupled DETF MEMS resonators observed at resonator 1 under two different conditions of electrical coupling (a) $4 \mathrm{~V}$ potential difference across the coupling gap (b) $8 \mathrm{~V}$ potential difference across the coupling gap.

TABLE I. SUMMARY OF EMPIRICAL RESULTS

\begin{tabular}{lcccc}
\hline Oscillator & Frequency $(\mathbf{H z})$ & Output power $(\mathbf{d B m})$ & \multicolumn{2}{c}{ Phase ratio (degrees) } \\
\cline { 4 - 5 } & & & Mean & Standard deviation \\
\hline Uncoupled Oscillator 1 & 219402.4 & -55.6 & 169.4 & 41.4 \\
Uncoupled Oscillator 2 & 219403.6 & -57.6 & 156.5 & 54.9 \\
Coupled Oscillator 1 & 219401.3 & -50.8 & 180.4 & 18.2 \\
Coupled Oscillator 2 & 219401.3 & -50.9 & 178.8 & 20.4 \\
\hline
\end{tabular}

designed structures.

The coupled response of the resonators is shown in Fig. 4(a) and (b) for two different coupling forces. In first case, the potential difference across the coupling gap is $4 \mathrm{~V}$ while it is $8 \mathrm{~V}$ in the second case. The coupled response is observed at resonator 1 . The lower frequency mode is found to be out-of-phase normal mode which occurs first, due to the negative value of the electrical coupling spring.

\section{IMPLEMENTATION OF ELECTRICALLY COUPLED OSCILLATORS}

Fig. 5 illustrates the block diagram representation for the coupled MEMS oscillators. The two DETF resonators are embedded within the feedback loop of two distinct oscillator circuits. Each oscillator comprises a transimpedance amplifier for converting the motional current to a voltage and to meet the loop gain requirement for sustained oscillation. This block is followed by a band pass filter which removes unwanted normal modes. The filter output is then fed to a comparator. The comparator acts as a hard voltage limiter and limits the effect of amplitude fluctuations in the feedback signal. For both oscillators, output signals are observed at the output of the transimpedance amplifier as shown in Fig. 5. The response of the above configuration is compared for four different cases and the results are tabulated in Table I.

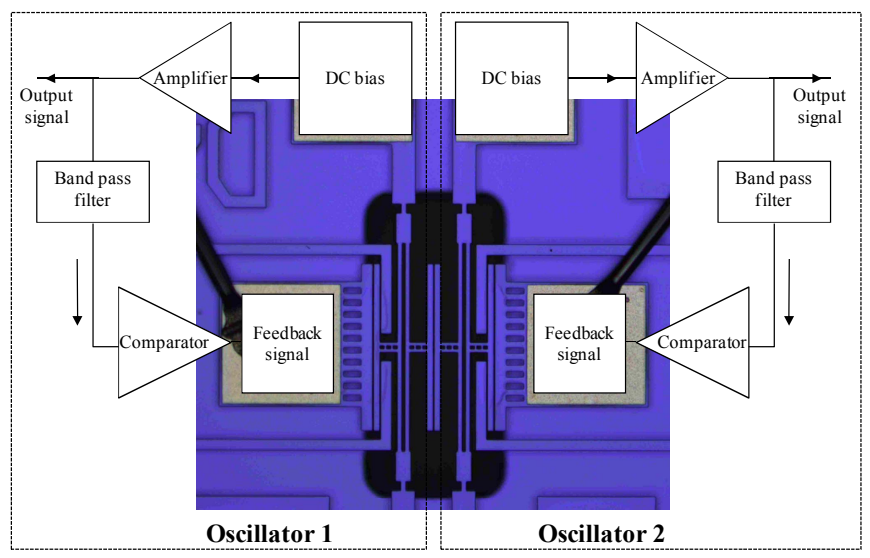

Figure 5. Schematic description of electrically coupled MEMS oscillators.

The first two cases are when both the oscillators are uncoupled (operated independently) and the next two cases when both are coupled (operated simultaneously). The observed output frequencies for oscillators 1 and 2 when operated independently, are $219402.4 \mathrm{~Hz}$ and $219403.6 \mathrm{~Hz}$. When operated simultaneously, the oscillators lock at a common oscillating frequency of $219401.3 \mathrm{~Hz}$ as shown in Fig. 6(a) and (b) respectively. 


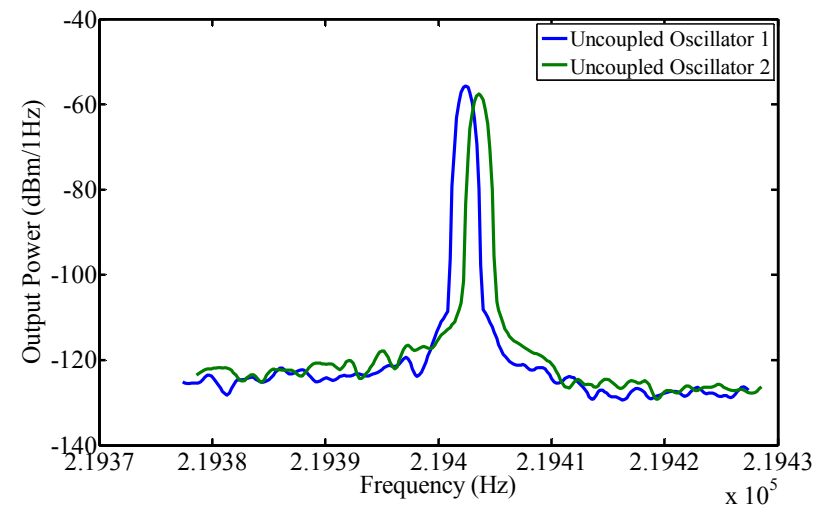

(a)

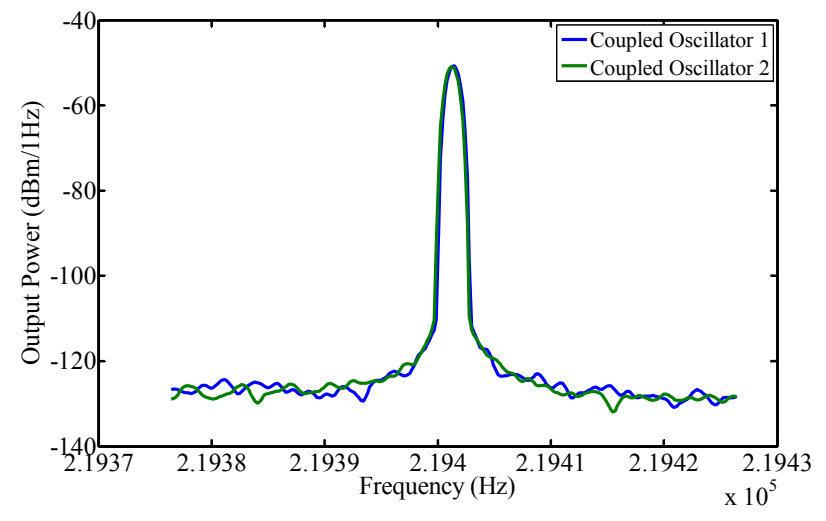

(b)

Figure 6. Output response of the MEMS oscillators compared before and after capacitive coupling (a) oscillators are uncoupled (b) oscillators are coupled.

In order to compare the oscillator response for uncoupled and coupled cases, a frequency normalized plot for oscillator 2 is shown in Fig. 7. A $6 \mathrm{dBm}$ gain in the output power and a clear reduction in noise floor can be observed for the case when the oscillators are coupled relative to the case when the two oscillators are uncoupled. Both oscillators describe the same general behaviour.

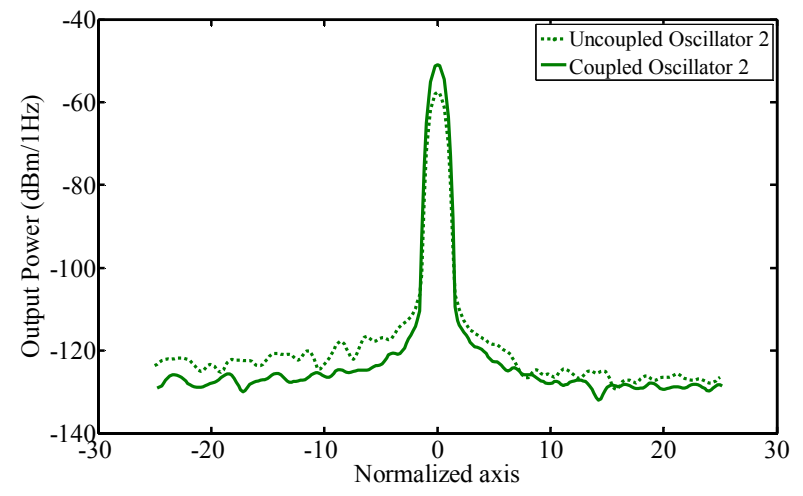

Figure 7. Frequency normalized response of oscillator 2 for uncoupled and coupled cases.

Table I summarizes the empirical results for the uncoupled and coupled MEMS oscillators. The phase ratio describes the relative phase of oscillator 1 with respect to oscillator 2. These measurements are done using a frequency counter (Agilent 53230A) at a sampling rate of $0.5 \mathrm{sec}$ for a total measurement time of $250 \mathrm{sec}$. For each case, the mean and standard deviation of the data are provided. From Table I, it can be seen that the coupled MEMS oscillators are oscillating with the same frequency. Moreover, the phase difference between the coupled oscillators is found to be approximately $180^{\circ}$ demonstrating locking to a common oscillation frequency with a constant phase difference. Moreover, the relative phase fluctuation is clearly reduced when the oscillators are coupled as compared to the case when the oscillators are uncoupled.
Measurements of phase noise and long-term oscillator stability before and after coupling are ongoing.

\section{CONCLUSION}

We propose the use of synchronization to enhance output signal power and enhance the signal-to-noise performance for coupled MEMS oscillators. The synchronized response of two coupled MEMS oscillators is demonstrated wherein the output amplitudes and signal-to-noise ratios of the coupled oscillators are clearly enhanced relative to the case before coupling. In addition, a reduction in relative phase fluctuations is observed for two coupled MEMS oscillators with the two oscillators locking $180^{\circ}$ out of phase. These initial results are promising and demonstrate that the phenomenon of synchronization can be extended to coupled MEMS oscillators resulting in improvements in oscillator noise performance. Future work includes more detailed investigations of noise and long-term stability in synchronized MEMS oscillators.

\section{ACKNOWLEDGMENT}

We thank Prof Jim Woodhouse for useful discussions. This work was funded in part by the UK-India Education and Research Initiative (UKIERI) grant SA06-250.

\section{REFERENCES}

[1] Y. W. Lin, S. S. Li, Z. Ren, and C. T.-C. Nguyen, "Low phase noise array-composite micromechanical wine-glass disk oscillator", in Tech. Dig., IEEE, IEDM, Washington DC, pp. 287-290, Dec 2005.

[2] J. R. Vig and Y. Kim, "Noise in microelectromechanical system resonators," IEEE Trans. Ultrason. Ferroelect. Freq. Contr., vol. 46, no. 6, pp. 1558-1565, Nov. 1999.

[3] B. Razavi, "A study of phase noise in CMOS oscillators," IEEE J. Solid-State Circuits, vol. 31, no. 3, pp. 331-343, Mar 1996.

[4] A. Pikovsky, M. Rosenblum, J. Kurths and R.C. Hilborn, Synchronization: a universal concept in nonlinear sciences. Cambridge University Press, 2001, pp. 1-3.

[5] M. Bennett, MF. Schatz and H. Rockwood and K. Wiesenfeld, "Huygens's clocks," Proc. R. Society A, pp. 563-579, Aug 2002. 
[6] P. Thiruvenkatanathan, J. Yan, J. Woodhouse, and A. A. Seshia, "Enhancing parametric sensitivity in electrically coupled MEMS resonators," J. Microlectromech. Syst., vol. 18, pp. 1077-1086, Oct 2009. 